\title{
GRB observations with the HETE soft X-ray cameras
}

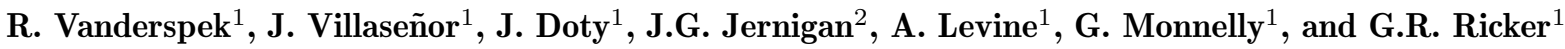 \\ 1 Center for Space Research, Massachusetts Institute of Technology, Cambridge, MA 02139, U.S.A. \\ 2 Space Sciences Laboratory, University of California, Berkeley, CA 94720, U.S.A.
}

Received January 21; accepted March 10, 1999

\begin{abstract}
The High Energy Transient Explorer, scheduled to be launched in late 1999 or early 2000, will carry a pair of Soft X-ray Cameras (SXC), capable of localizing the X-ray counterparts of GRBs with unprecedented precision. Each SXC consists of a pair of large-format CCDs behind a 1-D coded aperture: the two SXCs are oriented orthogonal to each other to permit two-dimensional image reconstruction. The SXCs on HETE will be able to localize bright $\mathrm{X}$-ray afterglows to $<20^{\prime \prime}$ precision within seconds of burst onset. The coordinates of detected bursts will be distributed immediately to ground-based observers and to the GCN via the HETE Burst Alert System.
\end{abstract}

Key words: gamma-ray: bursts — instrumentation

\section{Introduction}

The first High Energy Transient Experiment satellite was a multiwavelength mission built to detect and localize gamma-ray bursts (GRBs). It was equipped with NaI gamma-ray detectors (the French Gamma Telescope, or Fregate), two crossed one-dimensional X-ray coded-aperture detectors (the Wide-Field $X$-ray Monitor, or WXM; see Yoshida et al. 1999) and four near-UV-sensitive CCD cameras, which doubled as the spacecraft's star trackers. HETE was designed to detect and localize GRBs in real time and to send the coordinates of detected bursts to ground observers within seconds of burst onset for prompt followup observations. The first HETE satellite was launched in November 1996, but was not deployed in orbit due to a failure in the third stage of the Pegasus launcher.

The observations of GRBs by BeppoSAX in the months following the loss of HETE demonstrated the existence of X-ray afterglows in a large fraction of GRBs and also led to the discovery of faint $(m \sim 20-22)$ optical afterglows. Analyses of GRBs detected by Ginga (Strohmayer et al. 1998) discussed that year show that many GRBs emit a significant fraction of their total energy in photons with $E<10 \mathrm{keV}$. Thus, for the reflight of HETE (Ricker 1999), we have replaced two of the UV camera systems with two one-dimensional

Send offprint requests to: R. Vanderspek
CCD-based coded aperture systems sensitive to soft X-rays $(0.5-12 \mathrm{keV})$ : these are the Soft X-ray Cameras, or SXCs. (The other two UV cameras have been converted to standard CCD cameras sensitive to optical photons). In this paper, we review the details of the SXC instrumentation, flight operation, and sensitivity.

\section{SXC instrumentation}

Each SXC module consists of a pair of large-format CCDs behind a finely-ruled one-dimensional coded aperture; two such modules, with orthogonal localization capabilities, comprise the full SXC complement. An optical "boresight" CCD camera is comounted with each SXC module to provide an absolute reference system for detected X-ray transients. Details of the SXC X-ray and optical systems can be found in Table 1.

The mask elements (slits) are oriented parallel to the CCD columns, so the mask shadow patterns fall along CCD columns. The CCD is clocked in a fashion where every row of data read out is the sum of 100 rows on the detector (similar to "fast" mode on ASCA): the CCD thus acts as a one-dimensional detector. The positions of $\mathrm{X}$-ray sources in the field-of-view are calculated by crosscorrelating the mask pattern with a histogram of the number of events detected in each CCD column. The angular size of one cross-correlation element is $33^{\prime \prime}$.

The mask is an electroformed gold sheet $30 \mu \mathrm{m}$ thick, capable of blocking $99 \%$ of $14 \mathrm{keV}$ photons. The slits in the mask have widths which are integral multiples of $45 \mu \mathrm{m}$. The mask is covered by an optical blocking filter of $5000 \mathrm{~A}$ of polyimide and 1500A of aluminum.

The CCDs are frontside-illuminated MIT/Lincoln Laboratory CCID-20 devices. These CCDs share heritage with the ACIS CCDs being flown on the Chandra X-ray Observatory: they have good sensitivity to $500 \mathrm{eV}$ photons and have a demonstrated energy resolution of $150 \mathrm{eV}$ at $5.9 \mathrm{keV}$ when clocked in the manner described above. One of the two CCDs in each SXC module is covered with a $25 \mu \mathrm{m}$ sheet of beryllium as a hedge against the possibility of damage by micrometeorites.

In order to achieve good localization precision, we have placed stringent requirements on the manufacture and stability of the SXCs. The key requirements on the SXC 
Table 1. SXC instrumental characteristics

\begin{tabular}{ll}
\hline Characteristic & Value \\
\hline Number of cameras & $2($ one $X$, one $Y)$ \\
Module Dimensions & $10 \mathrm{~cm} \times 10 \mathrm{~cm} \times 10 \mathrm{~cm}$ \\
CCD Dimensions & $2048 \times 4096,15 \mu \mathrm{m} /$ pixel \\
Field of view & 0.92 steradian $(\mathrm{FWHM})$ \\
& 1.8 steradian $(\mathrm{FWZM})$ \\
Pixel angular size & $33^{\prime \prime}$ \\
Energy range & $0.5-12 \mathrm{keV}$ \\
Detector area & $75 \mathrm{~cm}^{2}$ (two cameras) \\
Mask open fraction & 0.2 \\
Minimum mask slit width & $45 \mu \mathrm{m}$ \\
Boresight camera FOV & $5.5^{\circ} \times 5.5^{\circ}$ \\
Boresight camera pixel size & $77^{\prime \prime}$ \\
\hline
\end{tabular}

hardware, which must be met over the full range of SXC operating temperature, are 1) that the mask slits are parallel to each other to within $5 \mu \mathrm{m}$ over the length of the mask, and 2) that the mask slits are parallel to the CCD columns to within $5 \mu \mathrm{m}$ over the length of the mask $(5 \mu \mathrm{m}$ skew adds $\sim 5^{\prime \prime}$ to the $1 \sigma$ uncertainty in the burst localization). Thermal and mechanical models show that the SXC hardware should be isothermal and sufficiently stable to meet these requirements, and tests with a prototype SXC have demonstrated the stability of the mechanical system over a wide range of temperatures.

\section{SXC flight operation}

During normal flight operations, the CCDs are read out in the "fast" mode described above. Each row of CCD data is examined for X-ray events (the method is very similar to the one used with the MIT-developed X-ray CCD instrument on the ASCA satellite), and a "running difference" correlation map is continuously calculated. This map is calculated as the difference between the correlation map using the last $N$ photons and one using $N$ photons detected $\sim 2 N$ photons earlier, where $N$ is typically 1000. This method has enormous advantages: first, the contribution of steady background sources is continuously subtracted, and, second, there is always a current "best answer". Thus, if a GRB is detected on board, any statistically-significant peak in the running-difference correlation map is likely to be due to an X-ray counterpart to the GRB.

If there is no statistically-significant peak in the crosscorrelation function, the SXC alone will not be able to provide a precise localization for the GRB. However, a peak which is not statistically significant when the entire correlation map is examined becomes more significant if only a fraction of the map containing that peak is examined. We plan to use localization information given by the WXM on board in real time to reduce the region of the correlation map examined: in this way, smaller peaks in the specified region may become statistically significant and give us a more precise localization.
The aspect of each SXC is measured using the boresight cameras comounted with the SXC CCDs. The boresight camera pixel size is $\sim 1^{\prime}$, but measurement of many stellar images allows the orientation to be calculated to a precision of a few arc-seconds. Since the boresight camera and SXC are co-mounted, we expect the relation between the SXC and optical coordinate systems to be highly stable.

\section{SXC sensitivity and localization precision}

A simplified expression for the signal-to-noise ratio for a coded-aperture system with minimal slant-ray blur (Doty 1988) is

$S / N=\frac{S}{\sqrt{S+\frac{f}{1-f} B}}$,

where $S$ is the source strength, $B$ is the contribution of the diffuse X-ray background, and $f$ is the open fraction of the aperture. We estimate that the sensitivity of a pair of SXC modules is $520 t^{-0.5} \mathrm{mCrab}$ for an $E^{-2}$ photon spectrum, where $t$ is the integration time in seconds. Because HETE is always anti-solar pointing, long exposures of detected burst sources will be possible. However, most X-ray afterglows decay much more quickly than $t^{-1 / 2}$. Given the time histories of afterglows seen to date, we realistically expect to be able to detect most afterglows up to $100-200$ seconds after burst onset.

The accuracy with which the SXC system can localize the X-ray counterpart to a GRB depends on both systematic and statistical errors. The statistical contribution to the localization precision can be estimated by the intrinsic pixel angular size $\left(33^{\prime \prime}\right)$ over the signal-to-noise ratio of the peak in the correlation map. The SXC aspect should be precise to $<5^{\prime \prime}$. Sources of systematic error, including the non-parallelism of the mask slits and the CCD columns and any deformations in the mask, should be less than $10^{\prime \prime}$. Using these estimates, the localization precision is strongly dominated by systematics: a $4 \sigma$ X-ray detection will be localized to $14^{\prime \prime}$, while a $10 \sigma$ burst will be localized to $11^{\prime \prime}$ ( $1 \sigma$ radius). There is a reasonable expectation that the mask/CCD alignment can be maintained to better than $5 \mu \mathrm{m}$, which would reduce that contribution to the blur budget to $\sim 5 \mu \mathrm{m}$ : the resulting localization precisions would then be $12^{\prime \prime}$ and $8^{\prime \prime}$, for the $4 \sigma$ and $10 \sigma$ cases.

Acknowledgements. The HETE program is supported by NASA Contract NASW-4690.

\section{References}

Doty J.P., 1988, in "X-Ray Instrumentation in Astronomy II", SPIE Vol. 982

Strohmayer T.E., et al., 1998, ApJ 500, 873

Ricker G.R., 1999 (these proceedings)

Yoshida A., et al., 1999 (these proceedings) 